\title{
Metacognitive interpersonal therapy in group: a feasibility study
}

\author{
Raffaele Popolo, ${ }^{1}$ Angus MacBeth, ${ }^{2}$ Stefano Brunello, ${ }^{3}$ Flaviano Canfora, ${ }^{4}$ Ercan Ozdemir, ${ }^{2}$ Daniela Rebecchi, ${ }^{3}$ \\ Cecilia Toselli, ${ }^{5}$ Gloria Venturelli, ${ }^{3}$ Giampaolo Salvatore, ${ }^{1,6}$ Giancarlo Dimaggio ${ }^{1}$
}

${ }^{1}$ Centro di Terapia Metacognitiva Interpersonale, Rome, Italy; ${ }^{2}$ University of Edinburgh, Edinburgh, Scotland, UK; ${ }^{3}$ Servizio Psicologia Clinica DSM, AUSL Modena, Modena, Italy; ${ }^{4}$ Servizio Adolescenti CEIS, Modena, Italy; ${ }^{5}$ Villa Igea, Modena, Italy; ${ }^{6}$ Scuola di Specializzazione "Humanitas", Rome, Italy

\begin{abstract}
Patients with personality disorders (PDs) other than borderline, with prominent features of social inhibition and over-regulation of emotions, are in need of specialized treatments. Individuals present with poor metacognition, that is the capacity to understand mental states and use psychological knowledge for the sake of purposeful problem solving; and are guided by maladaptive interpersonal schemas. We developed a short-term group intervention, Metacognitive Interpersonal Therapy in Groups (MIT-G), incorporating psychoeducational and experiential elements, to help these individuals become more aware of their drives when interacting with others; and to help them adopt more flexible behaviors via improvements in metacognition. We present results of an effectiveness study, evaluating whether we could replicate the initial positive results of our first pilot randomized controlled trial. Seventeen young adults outpatients with personality disorders were included in the 16 session program. Effect sizes were calculated for change from baseline to treatment end for the primary outcome, symptoms and functioning (Clinical Outcomes in Routine Evaluation Outcome Measure) and then for one putative mechanism of change - metacognition. Emotional dysregulation and alexithymia were also assessed. Qualitative evaluations of the acceptability and subjective impact of the treatment were also performed. MIT-G was acceptable to participants. There were medium to large magnitude changes from pre- to post- treatment on wellbeing, emotion dysregulation, alexithymia and metacognition. These gains were maintained at follow-up. There was evidence of clinically significant change on key variables. MIT$\mathrm{G}$ appears acceptable to patients, as evidenced by the absence of drop-out from treatment. In light of the positive outcomes of this study and the expanding evidence base, MIT-G is a candidate for dissemination and investigations in larger trials as a possible effective intervention for PDs characterized by tendencies to overcontrol.
\end{abstract}

Key words: Metacognition; Group; Psychotherapy; Personality disorder; Effectiveness.

Correspondence: Raffaele Popolo, Centro di Terapia Metacognitiva Interpersonale, Piazza dei Martiri di Belfiore 4, 00195 Rome, Italy. Tel./Fax: +39.644233878 .

E-mail: popoloraffaele@gmail.com

Citation: Popolo, R., MacBeth, A., Brunello, S., Canfora, F., Ozdemir, E., Rebecchi, D., ... Dimaggio, G. (2018). Metacognitive interpersonal therapy in group: a feasibility study. Research in Psychotherapy: Psychopathology, Process and Outcome, 21(3), 155163. doi: 10.4081 /ripppo. 2018.338

Contributions: the authors contributed equally.

Conflict of interest: the authors declare no potential conflict of interest.

Funding: none.

Received for publication: 10 September 2018.

Revision received: 7 December 2018.

Accepted for publication: 10 December 2018.

This work is licensed under a Creative Commons Attribution NonCommercial 4.0 License (CC BY-NC 4.0).

CCopyright R. Popolo et al., 2018

Licensee PAGEPress, Italy

Research in Psychotherapy:

Psychopathology, Process and Outcome 2018; 21:155-163

doi:10.4081/ripppo.2018.338

\section{Introduction}

Many treatments are available for Borderline Personality Disorders (BPDs), but only a few studies are available for other personality disorders (PDs), in particularly those characterised by social and emotional inhibition (Arnevik et al., 2009; Bamelis et al., 2014; Gullestad et al. 2012). The dearth of outcome studies for this population is a problem as the cumulative prevalence of these PDs and associate presentations are accompanied by complex symptoms and social problems (Karterud, Øien \& Pedersen, 2011; Soeteman, Verheul \& Busschbach, 2008). Recently, interest in these PDs is increasing, with authors consistently noting the need for specific treatments, which differ from those treatments usually adopted for BPD (Dixon-Gordon, Turner, \& Chapman, 2011). In particular, many note that these disorders are maintained by typical maladaptive interpersonal schemas, albeit named differently according to therapeutic orientation (Bowlby, 1969; Dimaggio, Montano, Popolo, \& Salvatore, 2013; Young, 1990). Therefore improvements require individuals to first be more flexible about their attributions regarding self and others in order to increase functioning and reduce distress.

One such treatment is Metacognitive Interpersonal Therapy (MIT). This was first developed for the whole 
range of PDs, (Dimaggio et al., 2007), then manualized with particular attention to the PDs introduced above, usually diagnosed as Avoidant, Dependent, Obsessive Compulsive, or Paranoid PDs, or PDs with covert narcissism and dependent and passive-aggressive traits. These PDs are also been defined as internalizing (Harford et al., 2013; Krueger, McGue, \& Iacino, 2001) or with prominent traits of detachment and negative affectivity (American Psychiatric Association, 2013). MIT has demonstrated effectiveness in a single case series of patients with PD (Dimaggio et al., 2017) and results have been replicated in a multiple-baseline single case series (Gordon-King, Schweitzer \& Dimaggio, 2017).

PDs can be understood as a pathology of social relationships, so if therapy is designed in order to make clients accept a group-based approach, such a format may be more ecologically valid and outcomes may be easier to translate into real life. For this reason and to increase economic viability (making the therapy more likely to be applied in public mental health settings) an adaptation, Metacognitive Interpersonal Therapy in Group (MIT-G) has been devised; (Popolo et al., 2018). MIT-G rests on a series of assumptions, particularly: i) PDs feature poor metacognition; and ii) humans are driven by a set of evolutionarily shaped motives. Providing patients with information about these motives helps them to quickly gain an understanding of what drives them to act, and develop an understanding of the cognitive and affective processes developing during interpersonal interactions. Patients also better understand better how their maladaptive interpersonal schemas develop around these motives. As an example - when driven by the social rank motive, a patient may have a dominant self-image of themselves as flawed, inferior and inept. Thus, they predict the other will criticize him or her, and accordingly the patient perceives their need to be appreciated has been frustrated.

\section{Metacognition}

We engage in metacognitive activity by identifying and understanding how we feel and what drives us to act, and by forming an integrated view of ourselves despite the various mental states continuously alternating in our minds. We identify the contents of experience on the basis of perceptual and linguistic cues, as well as using chains of inferences about mental functioning. For example, we can identify our emotional state by focusing on our bodily sensations. We also use metacognitive skills when trying to understand how others feel and the intentions that are likely guiding their behavior. For example, we can guess others' emotions by observing their facial expressions, posture and prosody. Lastly, metacognition includes the ability to use an understanding of mental states to manipulate and regulate them, which is metacognitive mastery. For instance, mastery may be evidenced by manipulating conditions in which we calm down, concentrate, relax or enjoy ourselves. Overall, metacognition refers to the com- bination of skills which enable humans to: identify mental states and ascribe them to themselves and others; think, reflect and reason about their own mental states (self-reflectivity) and think, reflect and reason about other's mental states (understanding others' minds); use the knowledge and reflections of their own and others' mental states to make decisions, solve problems or psychological and interpersonal conflicts, and master subjective suffering (mastery).

An individual's metacognitive skills can fluctuate as the quality of their relationships varies. In particular, in PDs metacognition greatly depends on levels of emotional arousal and on the quality of relationships (Fonagy, Gergely, Jurist, \& Target, 2002). For example, if a person perceives relational threats and expects the other to attack, abuse or dominate, he or she resorts to basic attributions, and easily think that the other's mind is inhabited by hostile or malicious intentions (Liotti \& Gilbert, 2011; Lysaker et al., 2011). Consequently, he or she will not be able to flexibly form ideas about what is passing though the mind of the other. In contrast, when the person is calm and relaxed, he or she will be more likely to read the mind of the other in more nuanced, realistic and context-specific way. Metacognition has been found to be impaired in the wide range of PD and associated to symptoms and interpersonal difficulties (Lysaker et al., 2014; Maillard et al., 2017; Moroni et al., 2016; Outcalt et al., 2016; Pellecchia et al., 2018; Semerari et al., 2014) and appears as growing during successful treatments (Carcione et al., 2011; Dimaggio, Vanheule, Lysaker, Carcione, \& Nicolò, 2009; Maillard et al., 2017; Popolo et al., 2018; Semerari et al., 2005).

\section{Interpersonal motives}

When individuals with PD are driven by specific personal motivations, they experience intense emotions, often negative, and correspondingly have difficulties recognizing what they feel, leading to difficulties in understanding the intentions of others and regulating one's own behavior on the basis of awareness of mental states (Liotti \& Gilbert, 2011). In the realm of social relationships humans are driven by goals they need to reach in order to feel safe, find their place in the society, and live a fully adapted and fulfilling life. In an evolutionary framework, the relevant goals human pursue are: social rank/competition, attachment, sexuality, group belonging or group inclusion, caregiving, cooperation with peers to reach shared goals and autonomous exploration of the environment (which includes curiosity over psychological states and processes) (Fassone et al., 2016; Gilbert, 1989; 2005; Ivaldi, 2016; Lichtenberg, Lachmann, \& Fosshage, 2016; Liotti \& Gilbert, 2011; Paanksepp, 1988).

Every motive has specific triggers and individuals experience a range of typical thoughts and affects depending on their prediction of whether the motive is fulfilled or 
unfulfilled. As an example, social rank becomes active in the presence of limited resources. In order to have access to these resources individuals enter into competition in order to establish priority of access. Typical emotions linked to this are: rage when one's own status is threatened; or pride and superiority when we evaluate that we have the status we want and think we deserve. Conversely when individuals consider they are inferior, they fear social judgement, experiencing shame, embarrassment and humiliation. Sadness is also relevant as an individual may think they have lost the chance to have fast track access to resources, and may predict that things will not change in the future.

\section{Development of metacognitive interpersonal therapy in group}

We hypothesized that metacognitive functioning could be effectively trained in a group setting, creating a seminaturalistic space where patients can practice these abilities by interacting with others in a protected area. In the course of exchanges with others, participants try to identify the elements that distinguish their interpersonal patterns, which are automatically activated and upon which they do not usually reason in real life. The feedback that they receive from the group enables them to evaluate their convictions and their behaviors according to a different perspective and not always in the usual way. The individual can then try to wonder about what others think and find out they may have beliefs or viewpoints different from those they anticipated. With these ideas, we developed MIT-G, with the goal of delivering an effective therapy at reduced cost.

MIT-G aims to improve metacognition so that participants can use a richer and fine grained understanding of mental states to regulate interpersonal interactions that develop when they are driven by relevant personal motives. For example, a young man desires to date a woman whom he very much likes, but he is blocked by a sense of confusion and agitation. He becomes unable to decode what is passing through the mind of the woman: Does she like him? Is she happy, upset, why is she blushing? In this state of confusion the young man is likely to resort to maladaptive schema-driven attributions: "She is not interested in me, I am disappointing her" (Dimaggio et al., 2013). MIT$G$ proceeds from the basis that the understanding of mental states can be taught and trained. Explaining the most important human motives driving human behavior to participants can help them to make sense of others' actions. Psychoeducation stimulates reflection in patients on their relational skills and coping with daily difficulties. These techniques come into play during the exercise of different metacognitive skills in the interpersonal exchange. In order to practice metacognitive skills we use role play as a technique that allows patients to respond to interpersonal difficulties in a contained safe environment. This helps stimulate a clearer understanding of their own men- tal states and correspondingly enables them to be more flexible and context-specific in ascribing mental states to the other. First, patients write an autobiographical episode concerning the motive presented during psychoeducation. The patient then role-plays the episode, and after the enactment a group discussion ensues, focused on understanding the mental states of the actors.

MIT-G was reported to be effective in a pilot randomized clinical trial (Popolo et al., 2018). Ten outpatients participated in MIT-G and pre-post outcomes were compared with 10 patients who received TAU. Drop-out rate was low (2 out of 10), and session attendance high (92.19\%). Participants in the MIT-G arm reported symptomatic and functional improvement equivalent to large effect sizes. Emotion dysregulation and alexithymia also significantly improved. Outcomes for metacognition also increased significantly from baseline to completion of treatment, particularly in the domains of self-awareness, decentration and mastery. Results were maintained at follow-up.

\section{Metacognitive interpersonal therapy in group (MIT-G) structure}

MIT-G comprises a short-term term manualized protocol, which includes psychoeducational and experiential aspects. The number of participants can vary from 5 to 10 to encourage active participation of all in a spirit of belonging and cooperation, and to ensure a participant's presence sufficient to form the group. The treatment is aimed at patients with PDs characterized by inhibited and over-regulated aspects (Dimaggio et al., 2018).

The MIT-G protocol consists of 16 weekly sessions lasting 2 hours each, during which basic interpersonal motives are described: i) agonistic, ii) affiliation $/ \mathrm{mem}$ bership, iii) attachment, iv) tracking, v) exploratory, vi) sexual and vii) cooperative. Two sessions are dedicated to each system (three for the cooperative one, which appears the most difficult to grasp). The final session is devoted to reflections on patients' experiences of the program, changes achieved and identifying further issues that they may wish to resolve in the future. Each group session is divided into two: a first psychoeducational phase, during which the interpersonal motive covered in the session is described; and an experiential phase involving a role play with subsequent discussion, where a story written by a patient about the motive presented is staged. The conductors of group are two MITcertified therapists.

Three individual sessions conducted by one of the group's facilitators are also arranged with each patient. The first session is held before the start of the group, the second in the course of the treatment and the third at the end of the group. During the first individual session, the therapist elicits narrative episodes related to the relational problems reported by the patient. At the end of the ses- 
sion, he or she shares a structured summary of the events following the typical structure of the interpersonal schemas (Dimaggio et al., 2013), including a general representation of it's functioning in the individuals relationships. The intermediate individual session is used to explore and clarify the group's progress from the perspective of the individual. It is then possible to evaluate elements of individual functioning or interpersonal difficulties that may impact upon the patient's participation in the group sessions. This reduces the chance of early interruptions in treatment and stimulates more active participation in the group itself. During the intermediate individual session, it is also possible to analyse any dysfunctional relational strategies that the patient has put in place during the group, locating these in relation to the possible activation of reconstructed schema. The last individual session aims to give the patient space to share his or her experience, deepening the understanding of what he or she has learnt about how to deal with interpersonal situations. Therapist and patient also devise a plan for how to promote further progress.

\section{Structure of sessions: psychoeducation}

Every session includes psychoeducation followed by experiential work. Psychoeducational elements are preceded by a warm up, during which participants can freely discuss general topics. For each motive, the conductors illustrate what it is, the conditions that activate and deactivate the motive, how relationships take place in this case, and the emotions that are activated with each motive. Thereafter, each motive is illustrated in two consecutive sessions, for about 10 minutes apiece. The conductors pay attention to the non-verbal communication amongst the participants, in order to be able to adapt their exposure to the group's requests/functioning, in order to foster a greater involvement.

After displaying the theoretical contents, the conductors present audio-video material, such as films, cartoons, clips, to illustrate the typical scenarios related to that motive. The aim is to facilitate rapid assimilation of the topics described through visual material. This material has the advantage of readily capturing the attention of participants, and serves to simplify the concepts described, using memorable, easily accessible scenes.

At the end of the video, which lasts about ten minutes, participants freely express their ideas on the topics covered. The purpose is to consolidate the knowledge of the motivation discussed and to clarify any doubts brought by the group. The video stimulates participants to identify processes involving the protagonists of the scenes observed, and primes the emergence of autobiographical memories. Each patient can express ideas about the video as well as on the material illustrated in the theoretical part, recalling personal associated relational experiences. The conductors try to summarise this material, linking to the theoretical arguments related to the motive.

\section{Structure of sessions: experiential phase}

After a short pause, the conductors ask the participants to write a short autobiographical episode involving protagonists in the interaction with one or more people; specifically, a scene in which they believe the motive described in the psychoeducational phase has been activated. The explicit purpose is to obtain a script to for the role play, first denoting the spatial (where) and temporal (when) boundaries in which the episode takes place. Participants then identify the characters present in the scene (age, sex, work, type of relationship that binds them to the author) and the sequence of actions succeeded. Among the written episodes, the therapists choose the one most relevant to motivation addressed and/or the motive identified by the participant as the most distressing element.

The purpose of role play is to transmit and encourage the acquisition of content from experience. The first role play of a specific motive focuses on the activation of selfreflective capacity. In the second session of the motive, the focus is on the promotion of a better understanding of others' minds and the elaboration of new, more effective strategies to manage interpersonal problems. The same scene is represented twice. In the first, the author represents himself and the episode described is reproduced more or less faithfully. In the second role play, instead, the roles are reversed and the patient now plays the role of the protagonist giving them the opportunity to add something personal to the script. The reversal of roles directly stimulates the theory of the mind and decentration skills of the actors, strengthening their awareness of their own internal states. During role play the participants are not forced to fully respect the written script. In the first session of each motive, the role play ends where the original episode ended. In the second session, instead, after the first enactment, the protagonist is asked to rescript the scene, introducing new attempts to solve the difficulties that emerged during the episode.

When role play is finished the conductors introduce the discussion, in which all the participants confront themselves on the experiences lived and the reflections made during the course and after the role play. In this phase, the conductors ask more specific questions to solicit the metacognitive functions: the aim being to get the individual to solicit these functions repeatedly in different sessions. The conductors propose simple questions to the group, and only in some cases to the single participant, thus guiding reflection a training the specific function for that session. The discussion does not follow a rigid sequence, allowing each individual to elaborate their own reflections according to their own times, and to feel free to express their own content as well as to comment upon others' content.

In the first session of each motive the conductors ask the participants to focus on theemotions they have experienced during enacting or watching the role play. After asking the participants to describe the emotional and cog- 
nitive components of their own internal states, the conductors help the group reflect on the subjective nature of their ideas, considering the possibility that these do not necessarily reflect what has happened, but that they can have different positions and perspectives. At this phase, we also investigate the strength of the conviction of the ideas reported by the participants in relation to the observed scene.

In the role play of the second session of each motive, the discussion is focused on specific relational strategies used by the actors during the representation. In the first part of the discussion, the aim is to stimulate the understanding of others' minds in participants, starting from the evaluation of motivations underlying the actors adoption of the relational modalities observed. During the discussion, the emergence of different opinions permits participants to enrich their repertoire of interpretations of others' behavior. At this stage there is also exploration of the differences between their style of response and that of the character, again emphasizing how different interpretations can be possible. In the second part of discussion, mastery skills are stimulated. First, conductors ask the main actor to describe his or her experience and to say whether he/she found the solution useful, and how difficult it was to adopt it. The conductors then ask observers whether they consider the new behavioural modalities staged by the actors to be effective, starting from the reactions they have elicited in the other characters in role play. Concurrently, the conductor offers feedback on more adaptive strategies, and highlight where strategies may be ineffectual or detrimental in everyday life.

\section{Study aims and hypotheses}

In this study, we wanted to further test the acceptability of MIT-G and its capacity to increase functioning and reduce distress. We also wanted to test the ecological validity of the approach in an implementation setting. MIT$\mathrm{G}$ groups were run in real-life conditions in a public mental health outpatient unit. We aimed to assess the magnitude of pre-post change on symptoms and functioning after receipt of MIT-G. In addition, we predicted a growth in metacognitive capacities as a potential mechanism of change.

\section{Design}

Young adults, aged between 16 and 25, and seeking treatment in Outpatient Mental Health Service in Modena, Italy, were screened for inclusion. Patients were referred by their treating clinicians, psychologists and psychiatrists working in this service between December 2016 and January 2017 on the basis of the presence of the following PDs: Avoidant, Dependent, Obsessive-Compulsive, Narcissistic, Paranoid, Passive-Aggressive and Depressive disorder, or PDNOS (meeting at least 10 SCID-II criteria). Exclusion criteria were: intellectual disability, organic brain disease or severe somatic disease impairing neuro- logical function, psychosis, bipolar I disorder or substance abuse severe enough to require specialized treatment. Patients hospitalized in the month before the beginning of recruitment were excluded, as were patients with prominent emotional dysregulation, intense suicidality or physical aggression towards others (due to overlap with Borderline PD). Substance abuse or drug intoxication were also exclusion criteria.

\section{Materials}

Symptoms and functioning were evaluated using the Clinical Outcomes in Routine Evaluation Outcome Measure (CORE-OM) (Evans et al., 2002), a 34-item self-report questionnaire. CORE-OM assesses problems and symptoms in four domains: subjective wellbeing, symptoms (anxiety, depression, somatic complaints, trauma-related); general, relational and social functioning, and risk (aggression towards self, e.g. suicidality, or towards others, e.g. aggression). Reliability for total score ranged from $\alpha=.91$ pre-treatment to $\alpha=.97$ at follow-up. Subscale reliability was high at all timepoints, with the exception of pre-treatment wellbeing $\alpha=.44$ and risk $\alpha=.63$.

Metacognition was assessed with the Metacognition Assessment Scale-Abbreviated (MAS-A) (Lysaker et al., 2005) a rating scale adapted from the MAS (Semerari et al., 2003). Scores are assigned after reading transcripts from the Indiana Interview for Psychiatric Illness (Lysaker et al., 2002). MAS-A evaluates 4 domains of metacognition: Self-reflectivity, Awareness of the Mind of the Other, Decentration and Mastery.

Emotional dysregulation was assessed with the Difficulties in Emotion regulation scales (DERS) (Gratz \& Roemer, 2004; Giromini et al., 2012), a self-report questionnaire. Here, we used the total score only. Reliability for total score ranged from $\alpha=.92$ pre-treatment to $\alpha=.80$ at follow-up.

Alexithymia was assessed with the Toronto Alexithymia Scale-20 (TAS-20) (Bagby et al., 1994 a; 1994b), a 20-item self-report measure. TAS-20 has 3 subscales: Difficulty Describing Feelings subscale, Difficulty Identifying Feeling subscale and Externally-Oriented Thinking subscale. Reliability for total score ranged from $\alpha=.86$ pre-treatment to $\alpha=.91$ at follow-up. Subscale reliability was acceptable at all timepoints $(\alpha .73-.88)$.

\section{Procedures}

Seventeen patients met inclusion criteria and were referred to 2 MIT-G groups (7 and 10 participants respectively). While undergoing MIT-G all patients continued TAU, which consisted of psychiatric medication monitoring (as required), supportive counselling and rehabilitation. The conductors, different in the two groups, carried out a specific training on MIT-G performed in their unit. During program delivery, therapists were supervised by one of the two developers of MIT-G (RP) for 1.5 hours per fortnight. Assessments of symptoms, functioning and psychological 
processes were conducted at baseline and repeated for both groups at termination and at a 3-months follow-up. Psychologists performing assessments were independent of group facilitators and therapists were blinded to outcome and process measures until after follow-up.

\section{Statistical analyses}

Descriptive statistics were calculated to examine demographic and clinical characteristics. Shapiro- Wilk's test was used to assess normality of the distributions of the pretreatment measures. Results indicated that the CORE-OM's Risk dimension $(\mathrm{P}<.05)$, and MAS's Self-Reflectivity $(\mathrm{P}<.001)$ and Mastery $(\mathrm{P}<.05)$ dimensions were significantly different from the normal distribution. Paired sample t-tests were calculated to examine whether individuals displayed significant improvements as measured by the outcome variables. All tests were two-tailed, with an alpha level of 0.05. Bootstrapped confidence intervals for mean change were created using 1000 resamples. Effect size was estimated using Cohen's $d$ where effect sizes of 0.2 are considered small, 0.5-0.6 medium, and $\geq 0.80$ large. Data analyses were carried out using SPSS for Windows, Version 24.0. To estimate clinically relevant changes across treatment, reliable change indices were calculated using the Leeds Reliable Change index calculator (Morley \& Dowzer, 2014). Appropriate reference groups were used to generate reliability data for CORE-OM (Evans et al., 2002), and DERS (Giromini et al., 2012).

\section{Results}

We recruited 17 participants who met inclusion criteria with an average age of 19 years (range $=16-23$ ). The sample was predominantly female (83\%) and the majority of participants were students $(n=15)$, with one unemployed individual and one in employment. The first group consisted of seven patients; six concluded treatment and one dropped-out at the tenth session, having exceeded the maximum number of absences indicated in the treatment protocol (three non-attendances). Of the 6 completers, attendance was very good, participants completed on average $>14$ of the 16 scheduled sessions (Attendance rate $=88.54 \%$ ). The second group consisted of ten patients; of these, nine ended the treatment and 1 dropped-out at the fourth session. The patient who interrupted treatment early, lived in the province, and had difficulty in reaching the service regularly, without being be accompanied by his/her parents. Of the 9 completers, attendance was very good, with participants completing on average $>14$ of the 16 scheduled sessions (Attendance rate $=90.28 \%$ ).

Outcomes and changes as a function of treatment are displayed in Table 1. From pre-post treatment participants improved in terms of CORE Wellbeing, equivalent to a large effect size (mean difference $=10.9,95 \% \mathrm{CI}=4.82$ to 15.71; $\mathrm{P}=.009 ; \mathrm{d}=.91)$. Participants also improved on
DERS total, equivalent to a large effect size (mean difference $=17,95 \% \mathrm{CI}=3.84$ to $28.41 ; \mathrm{P}=.034 ; \mathrm{d}=.74$ ), as well as on the Impulse Control Difficulty (mean difference $=3.75,95 \% \mathrm{CI}=1.25$ to $6.25 ; \mathrm{P}=.017 ; \mathrm{d}=.82)$ and Lack of Emotional Awareness (mean difference $=3,95 \% \mathrm{CI}=.83$ to $4.92 ; \mathrm{P}=.022 ; \mathrm{d}=.85$ ) subscales. Participants' total score on TAS (mean difference $=9.43,95 \% \mathrm{CI}=3.79$ to 15.43 ; $\mathrm{P}=.004 ; \mathrm{d}=.82$ ) significantly reduced between baseline and post-treatment, and also on the Difficulty Describing Feelings (mean difference $=3.64,95 \% \mathrm{CI}=1.14$ to $8.21 ; \mathrm{P}=.021$; $\mathrm{d}=.72$ ) and Difficulty Identifying Feelings (mean difference $=4.1,95 \% \mathrm{CI}=1.14$ to $7 ; \mathrm{P}=.022 ; \mathrm{d}=0.7$ ) subscales.

Between baseline and follow-up, paired samples ttests indicated that CORE-OM Total scores (without Risk items) showed significant improvement equivalent to a medium effect size (mean difference $=5.03095 \% \mathrm{CI}=.98$ to $8.96 ; \mathrm{P}=.044 ; \mathrm{d}=.66$ ); and the Wellbeing subscale also improved with a large magnitude effect (mean difference $=7.08,095 \% \mathrm{CI}=2.92$ to $11.25 ; \mathrm{P}=.009 ; \mathrm{d}=.93$ ).

For emotion regulation, DERS total score showed a large magnitude reduction (mean difference $=19.27,95 \%$ $\mathrm{CI}=4.55$ to $30.81 ; \mathrm{P}=.02 ; \mathrm{d}=.88$ ); mirrored by large reductions in the Lack of Emotional Awareness and Lack of Emotional Clarity subscales (respectively, mean difference $=4.54,95 \% \mathrm{CI}=2.54$ to $6.82 ; \mathrm{P}=.022 ; \mathrm{d}=1.17$; and mean difference $=4.64,95 \% \mathrm{CI}=1.27$ to $7.91 ; \mathrm{P}=.036$; $\mathrm{d}=0.8$ ). For alexithymia, there was a very large magnitude reduction in TAS total score (mean difference $=8.17,95 \%$ $\mathrm{CI}=5.67$ to $10.42 ; \mathrm{P}=.001 ; \mathrm{d}=1.93$ ); and large reductions in the Difficulty Describing Feelings (mean difference $=1.83$, $95 \% \mathrm{CI}=.58$ to $3.08 ; \mathrm{P}=.029 ; \mathrm{d}=.81$ ) and Difficulty Identifying Feelings subscales (mean difference $=4.25,95 \% \mathrm{CI}=2$ to $6.33 ; \mathrm{P}=.003 ; \mathrm{d}=1.08$ ). There were no significant differences between post-treatment and follow up measures, indicating no decrease in gains made during treatment.

With regard to potential mechanisms of change, participants' metacognitive skills improved in all MAS-A subscales, with large effect size. In particular, there were significant differences between baseline and post-treatment values for Self-Reflectivity (mean difference $=-.87$, $95 \% \mathrm{CI}=-1.2$ to $-.57 ; \mathrm{P}=.001 ; \mathrm{d}=1.35)$, Awareness of the Mind of the Other (mean difference $=-.5,95 \% \mathrm{CI}=-.8$ to $.23 ; \mathrm{P}=.017 ; \mathrm{d}=.88$ ), Decentration (mean difference $=-.53$, $95 \% \mathrm{CI}=-.9$ to $-.17 ; \mathrm{P}=.03 ; \mathrm{d}=.71$ ), and Mastery (mean difference $=-.83,95 \% \mathrm{CI}=-1.26$ to $-.4 ; \mathrm{P}=.015 ; \mathrm{d}=.97)$.

Finally, outcome variables were further analysed using the Reliable Clinical Index and Clinically Significant Change (CSC) methods. For CORE-OM Wellbeing, between baseline and post-treatment outcomes, 6 participants showed reliable change, 5 of whom met the criterion for clinically significant change $(n=14)$. Analyses of DERS total score revealed clinically significant change for 1 participant and 6 participants showed reliable change $(\mathrm{n}=12)$. In terms of subscales, for the DERS' Impulse Control Difficulties subscale, no participants showed $\mathrm{CSC}$, while 3 showed reliable change $(\mathrm{n}=15)$. Out of 15 
participants, 3 showed reliable change on DERS's Lack of Emotional Awareness subscale, among whom 1 participant met the CSC criterion.

\section{Discussion}

Patients with over-controlled, socially and emotionally inhibited patients require cost-effective specialized treatments. MIT-G is a candidate treatment based on promising results from a pilot randomized controlled trial (RCT) (Popolo et al., 2018). In this feasibility study, we aimed at replicating the results of the former trial in ecologically valid conditions, in this case implemented as routine clinical practice via an outpatient public service. In line with expectations, patients adherence to therapy was high, with 15 out of 17 patient completing treatment. Importantly, results from the first trial (Popolo et al., 2018) were successfully replicated. Primary outcomes were symptoms and functioning, which improved with medium to large effect sizes. The secondary outcomes were emotion unawareness and dysregulation, which also improved with medium to large effects, which were particularly striking for alexithymia. As regards metacognition as the hypothesized mechanism of change (Semerari et al., 2003), improvent was evident. In summary, patients in this sample had better functioning and less symptoms post treatment, which was accompanied by an increased capacity to understand mental states and to use mentalistic knowledge for the sake of purposeful regulation of both emotions and social interactions. Importantly, gains were retained at follow-up.

Results encourage further testing of MIT-G in larger samples under methodologically robust RCT conditions. Equally, the results also support the potential for roll-out of MIT-G as a treatment for avoidant and emotionally inhibited PDs in routine clinical settings, potentially accompanied by implementation science-derived routine outcome evaluation.

Table 1. Changes in symptoms and functioning across timepoints.

\begin{tabular}{|c|c|c|c|c|c|}
\hline Measure & $\begin{array}{c}\text { Baseline } \\
\text { Mean (SD) }\end{array}$ & $\begin{array}{l}\text { Post-treatment } \\
\text { Mean (SD) }\end{array}$ & $\begin{array}{l}\text { Follow-up } \\
\text { Mean (SD) }\end{array}$ & $\begin{array}{c}\text { Effect size baseline } \\
\text { d }\end{array}$ & $\begin{array}{c}\text { Effect size follow-up } \\
\text { d }\end{array}$ \\
\hline CORE-OM Total & $17.66(5.44)$ & $12.98(8.17)$ & $13.72(8.38)$ & 0.46 & 0.59 \\
\hline Wellbeing & $25.89(5.33)$ & $15.89(9.43)^{\mathrm{a}}$ & $18.54(8.21)^{\mathrm{b}}$ & 0.91 & 0.93 \\
\hline Problems & $21.13(7.92)$ & $15.89(8.45)$ & $15.97(10.1)$ & 0.41 & 0.61 \\
\hline Functioning & $18.03(6.22)$ & $12.97(9.46)$ & $13.75(8.52)$ & 0.52 & 0.63 \\
\hline Risk & $4.52(4.82)$ & $5.23(9.51)$ & $5(8.4)$ & 0.07 & 0.14 \\
\hline Total without Risk & $20.48(5.98)$ & $14.64(8.27)$ & $15.59(8.88)^{\mathrm{b}}$ & 0.56 & 0.66 \\
\hline DERS Total & $107.6(24.1)$ & $90.66(23.6)^{\mathrm{a}}$ & $88.1(13.44)^{\mathrm{b}}$ & 0.74 & 0.88 \\
\hline NonAccept & $16.08(6.69)$ & $12.5(4.58)$ & $13.54(4.45)$ & 0.53 & 0.5 \\
\hline Goals & $17.5(4.52)$ & $16.5(4.23)$ & $16.36(4.17)$ & 0.25 & 0.41 \\
\hline Impulse & $17.08(5.74)$ & $13.33(3.79)^{\mathrm{a}}$ & $14.81(3.6)$ & 0.82 & 0.27 \\
\hline Aware & $17.5(4.31)$ & $14.5(4.85)^{\mathrm{a}}$ & $12.81(4.6)^{\mathrm{b}}$ & 0.85 & 1.17 \\
\hline Strategies & $23.83(7.1)$ & $20.91(7.47)$ & $19.27(3.97)$ & 0.4 & 0.63 \\
\hline Clarity & $15.66(5.22)$ & $12.91(4.77)$ & $11.27(2.79)^{\mathrm{b}}$ & 0.44 & 0.8 \\
\hline \multicolumn{6}{|l|}{ MAS } \\
\hline Self-Reflectivity & $4.2(1.3)$ & $5.06(1.08)^{\mathrm{a}}$ & - & 1.35 & - \\
\hline Others & $3.4(1.02)$ & $3.9(0.78)^{\mathrm{a}}$ & - & 0.88 & - \\
\hline Decentration & $1.66(0.89)$ & $1.7(0.72)^{\mathrm{a}}$ & - & 0.71 & - \\
\hline Mastery & $3.33(1.12)$ & $4.16(1.2)^{\mathrm{a}}$ & - & 0.97 & - \\
\hline TAS Total & $55.78(12.7)$ & $46.35(14.7)^{\mathrm{a}}$ & $47.5(14.96)^{b}$ & 0.82 & 1.93 \\
\hline Describing Feelings & $16.42(4.36)$ & $12.78(5.29)^{\mathrm{a}}$ & $14.25(4.99)^{\mathrm{b}}$ & 0.72 & 0.81 \\
\hline Identifying Feelings & $19.64(5.34)$ & $15.57(5.34)^{\mathrm{a}}$ & $16(6.57)^{\mathrm{b}}$ & 0.7 & 1.08 \\
\hline Thinking & $19.71(5.73)$ & $18.28(5.84)$ & $17.25(5.61)$ & 0.45 & 0.52 \\
\hline
\end{tabular}

CORE-OM, Clinical Outcomes in Routine Evaluation Outcome Measure; DERS, Difficulties in Emotion regulation scales; MAS, Metacognition Assessment Scale; TAS, Toronto Alexithymia

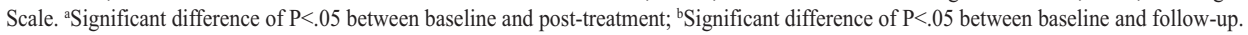




\section{Limitations}

There were a number of limitations to our study. First, given that both the current sample and the earlier RCT (Popolo et al., 2018) were comprised of young adults, results cannot be generalized to other age groups, in particular older patients. Another limitation was the absence of a control group, which limits our scope to establish whether change was caused by this specific program or was simply due to being included in a structured therapy program. Interpersonal functioning, which is the core feature of PD was not assessed. The relatively short duration of follow-up also leaves us unable to comment on whether results are sustained over time.

Finally, we did not assess changes in employment and work functioning, although we acknowledge these are key aspects of social adaptation in young adults.

\section{Conclusions}

Patients with PDs characterized by prominent features of social inhibition require specialized treatment. There is also a pressing need to deliver treatments with sustainable costs ad with ecological validity, with the hope that patients can generalize therapeutic gains to the 'real world'. MIT-G was developed with these goals, and in the current study we showed that it can be successfully applied as part of routine treatment delivery in a public mental health service. Acceptability was high with as evident from the low number of drop-outs (2 out of 17), and there was evidence of broad positive good outcomes in symptoms and functioning. Furthermore, participants emotion awareness and regulation improved over treatment, as well as their capacity to be aware of mental states and use psychological knowledge for the sake of purposeful problem solving. With replication and using methodologically rigorous clinical trials, MIT-G may be a promising effective and well accepted option for patients with PD.

\section{References}

American Psychiatric Association - APA (2013). Diagnostic and statistical manual of mental disorders, 5th ed. (DSM-5). Washington, DC: American Psychiatric Publishing.

Arnevik, E., Wilberg, T., Urnes, O., Johansen, M., Monsen, J.T., \& Karterud, S. (2009). Psychotherapy for personality disorders: short-term day hospital psychotherapy versus outpatient individual therapy - a randomized controlled study. European Psychiatry, 24(2), 71-78. doi:10.1016/j.eurpsy.2008.09.004.

Bagby, R.M., Parker, J.D.A., \& Taylor, G.J. (1994a). The Twenty - item Toronto Alexithymia Scale-I. Item selection and cross validation of the factor structure. Journal of Psychosomatic Research, 38, 23-32. doi:10.1016/0022-3999(94)90005-1

Bagby, R.M., Parker, J.D.A. \& Taylor, G.J. (1994b). The Twenty-item Toronto Alexithymia Scale-II. Convergent, discriminant and concurrent validity. Journal of Psychosomatic Research, 38, 33-40.
Bamelis, L.L.M., Evers, S.M.A.A., Spinhoven, P. \& Arntz, A. (2014). The results of a multicenter randomized controlled trial on the clinical effectiveness of schema therapy for personality disorders. American Journal of Psychiatry, 171, 305-322. doi:10.1176/appi.ajp.2013.12040518

Bowlby, J. (1969). Attachment and loss, Vol. 1: Attachment. New York: Basic Books.

Carcione, A., Semerari, A., Nicolò, G., Pedone, R., Popolo, R., Conti, L., ... \& Dimaggio, G. (2011). Metacognitive mastery dysfunctions in personality disorder psychotherapy. Psychiatry Research, 190, 60-71.

Dimaggio, G., MacBeth, A., Popolo, R., Salvatore, G., Perrini, F., Raouna, A., ... \& Montano, A. (2018). The problem of overcontrol: Perfectionism and emotional inhibition as predictors of personality disorder. Comprehensive Psychiatry. [Epub Ahead of print] doi:10.1016/j.compp sych.2018.03.005

Dimaggio G., Montano A., Popolo R., \& Salvatore G. (2013). Metacognitive interpersonal therapy for personality disorders: A treatment manual. London: Routledge.

Dimaggio, G., Procacci, M., Nicolò, G., Popolo, R., Semerari, A., Carcione, A. \& Lysaker, P.H. (2007). Poor Metacognition in narcissistic and avoidant personality disorders: analysis of four psychotherapy patients. Clinical Psychology and Psychotherapy, 14, 386-401. doi:10.1002/cpp.541

Dimaggio, G., Salvatore, G., MacBeth, A., Ottavi, P., Buonocore, L., \& Popolo R. (2017). Metacognitive interpersonal therapy for personality disorders: a case study series. Journal of Contemporary Psychotherapy, 47, 11-21. doi: 10.1007/s10879-016-9342-7

Dimaggio, G., Vanheule, S., Lysaker, P.H., Carcione, A., \& Nicolò, G. (2009). Impaired self-reflection in psychiatric disorders among adults: a proposal for the existence of a network of semi-independent functions. Consciousness \& Cognition, 18, 653-664. doi:10.1016/j.concog.2009.06.003

Dixon-Gordon, K., Turner, B. J., \& Chapman, A. L. (2011). Psychotherapy for personality disorders. International Review of Psychiatry, 23(3), 282-302. doi:10.3109/09540261.2011. 586992

Evans, C., Connell, J., Barkham, M., Margison, F., McGrath, G., Mellor-Clark, J., \& Audin, K. (2002). Towards a standardised brief outcome measure: psychometric properties and utility of the CORE-OM. British Journal of Psychiatry, 180, 51-60.

Fassone, G., Lo Reto, F., Foggetti, P., Santomassimo, C., D’Onofrio, M. R., Ivaldi, A., ... \& Picardi, A. (2016). A content validity study of AIMIT (Assessing Interpersonal Motivation in Transcripts). Clinical Psychology \& Psychotherapy, 23, 319-328. doi:10.1002/cpp.1960

Fonagy, P., Gergely, G., Jurist, E.L., \& Target, M. (2002). [Regolazione affettiva, mentalizzazione e sviluppo del sé]. Milan, Italy: Raffaello Cortina.

Gilbert, P. (1989). Human nature and suffering. London/New York: Psychology Press/Guilford Press.

Gilbert, P. (2005). Compassion and cruelty: a biopsychosocial approach. In Gilbert, P., Ed. Compassion: conceptualisations, research and use in psychotherapy (pp. 9-74). London, UK: Routledge.

Giromini, L., Velotti, P., de Campora, G., Bonalume, L., \& Zavattini G., C. (2012). Cultural adaptation of the difficulties in emotion regulation scale: reliability and validity of an Italian version. Journal of Clinical Psychology, 68(9), 9891007. doi:10.1002/jclp. 21876

Gordon-King, K., Schweitzer, R. D., \& Dimaggio, G. (2017). 
Behavioural activation in the treatment of metacognitive dysfunctions in inhibited-type personality disorders: a case study. Psychotherapy, 54, 252-259. doi:10.1037/pst0000120

Gratz, K. L., \& Roemer, L. (2004). Multidimensional assessment of emotion regulation and dysregulation: development, factor structure, and initial validation of the difficulties in emotion regulation scale. Journal of Psychopathology \& Behavioral Assessment, 26, 1, 41-54.

Gullestad, F. S., Wilberg, T., Klungsøyr, O., Johansen, M. S., Urnes, O., \& Karterud, S. (2012). Is treatment in a day hospital step-down program superior to outpatient individual psychotherapy for patients with personality disorders? 36 months follow-up of a randomized clinical trial comparing different treatment modalities. Psychotherapy Research, 22(4), 426-441. doi:10.1080/10503307.2012.662608

Harford, T. C., Chen, C. M., Saha, T. D., Smith, S. M., Ruan, W. J., \& Grant, B. F. (2013). DSM-IV personality disorders and associations with externalizing and internalizing disorders: Results from the National Epidemiologic Survey on Alcohol and Related Conditions. Journal of Psychiatric Research, 47, 11, 1708-1716.

Ivaldi, A. (2016). Treating dissociative and personality disorders: a motivational systems approach to theory and treatment. London: Routledge.

Karterud, S., Øien, M., \& Pedersen, G. (2011). Validity aspects of the diagnostic and statistical manual of mental disorders, fourth edition, narcissistic personality disorder construct. Comprehensive Psychiatry, 52(5), 517-526. doi:10.1016/j. comppsych.2010.11.001

Krueger, R.F., McGue, M., \& Iacono, W.G. (2001). The higherorder structure of common DSM mental disorders: internalization, externalization, and their connections to personality. Personality and Individual Differences, 30(7), 1245-1259.

Lichtenberg, J. D., Lachmann, F. M., \& Fosshage, J. L. (2016). Self and motivational systems: towards a theory of psychoanalytic technique. New York: Routledge.

Liotti, G. \& Gilbert, P. (2011). Mentalizing, motivation, and social mentalities: theoretical considerations and implications for psychotherapy. Psychology and Psychotherapy: Theory, Research and Practice, 84, 9-25. doi:10.1348/ 147608310X520094

Lysaker, P. H., Carcione, A., Dimaggio, G., Johannesen, J. K., Nicolò, G., Procacci, M., \& Semerari, A. (2005). Metacognition amidst narratives of self and illness in schizophrenia: Associations with insight, neurocognition, symptom and function. Acta Psychiatrica Scandinavica, 112, 64-71. doi:10.1111/j.1600-0447.2005.00514.x

Lysaker, P. H., Clements, C. A., Plascak-Hallberg, C. D., Knipscheer, S. J., \& Wright, D. E. (2002). Insight and personal narratives of illness in schizophrenia. Psychiatry, 65,197-206.

Lysaker, P.H., Gumley, A., Brune, M., Vanheule, S., Buck, K.D., \& Dimaggio, G. (2011). Deficits in the ability to recognize one's own affects and those of others: associations with neurocognition, symptoms and sexual trauma among persons with schizophrenia. Consciousness \& Cognition, 20, 11831192. doi:10.1016/j.concog.2010.12.018

Lysaker, P.H., Olesek, K., Buck, K., Leonhardt, B., Vohs, J., Dimaggio, G., Popolo, R. \& Outcalt, J. (2014). Metacognitive mastery moderates the relationship of alexithymia with cluster $\mathrm{C}$ personality disorder traits in adults with substance use disorders. Addictive Behaviors, 39, 558-561. doi:10.1016/ j.addbeh.2013.11.007

Maillard, P., Dimaggio, G., de Roten, Y., Despland, J-N., \& Kramer, U. (2017). Metacognitive processes and symptom change in a short-term treatment for borderline personality disorder: a pilot study. Journal of Psychotherapy Integration, 27(4), 445-459. doi:10.1037/int0000090

Morley, S. \& Dowzer, C.N. (2014). Manual for the Leeds Reliable Change Indicator: Simple Excel(tm) applications for the analysis of individual patient and group data. Leeds, UK: University of Leeds.

Moroni, F., Procacci, M., Pellecchia, G., Semerari, A., Nicolò, G., Carcione, A., Pedone, R., \& Colle, L. (2016). Mindreading dysfunction in avoidant personality disorder compared with other personality disorders. Journal of Nervous and Mental Diseases, 204(10), 752-757.

Outcalt, J., Dimaggio, G., Popolo, R., Olesek, K. L., Buck, K., \& Lysaker, P. H. (2016). Metacognition moderates the relationship of disturbances in attachment with severity of borderline personality disorder among individuals in treatment for substance use disorders. Comprehensive Psychiatry, 64, 22-28. doi:10.1016/j.comppsych.2015.10.002

Panksepp, J. (1998). Affective neuroscience: the foundations of human and animal emotions. Oxford: Oxford University Press.

Pellecchia, G., Moroni, F., Colle, L., Semerari, A., Carcione, A., Fera, T., .. \& Procacci, M. (2018). Avoidant personality disorder and social phobia: Does mindread- ing make the difference? Comprehensive Psychiatry, 80, 163-169. doi:10.1016/j.comppsych.2017.09.011

Popolo, R., MacBeth, A., Canfora, F., Rebecchi, D., Toselli, C., Salvatore, G., \& Dimaggio, G. (2018). Metacognitive interpersonal therapy in group (MIT-G) for young adults personality disorders. A pilot randomized controlled trial. Psychology and Psychotherapy: Theory, Research \& Practice. [Epub Ahead of print] doi:10.1111/papt.12182

Semerari, A., Carcione, A., Dimaggio, G., Falcone, M., Nicolò, G., Procacci, M., \& Alleva, G. (2003). How to evaluate metacognitive functioning in psychotherapy? the metacognition assessment scale and its applications. Clinical Psychology and Psychotherapy, 10, 238-261. doi: 10.1002/ cpp. 362

Semerari, A., Colle, L., Pellecchia, G., Buccione, I., Carcione, A., Dimaggio, G., ... \& Pedone, R. (2014). Metacognition: severity and styles in Personality Disorders. Journal of Personality Disorders, 28, 751-766.

Semerari, A., Dimaggio, G., Nicolò, G., Pedone, R., Procacci, M., \& Carcione, A. (2005). Metarepresentative functions in borderline personality disorders. Journal of Personality Disorders, 19, 690-710.

Soeteman, D. I., Verheul, R., \& Busschbach, J. J. (2008). The burden of disease in personality disorders: diagnosis-specific quality of life. Journal of Personality Disorders, 22(3), 259268. doi:10.1521/pedi.2008.22.3.259

Young, J. E. (1990). Cognitive therapy for personality disorders. A schema-focused approach. Sarasota, FL: Professional Resource Exchange, Inc. 